\title{
Simulation of a Tornadoes as a Particular Case of an Attractor-repeller Pairs
}

\author{
Giménez Lorente Araceli \\ School of Art and Design, Institut Superior D'Ensenyances Artistiques (ISEACV), Spain
}

Copyright $(\mathcal{C} 2015$ by authors, all rights reserved. Authors agree that this article remains permanently open access under the terms of the Creative Commons Attribution License 4.0 International License

\begin{abstract}
The attractor-repeller pairs are binary systems which are stable systems in termo-dynamic equilibrium, and one of the leaders we have in Nature to understand Tornadoes. A mathematical model of a tornado is presented, within a model of chaos theory, where two complementary fractals are combined to understand this natural phenomenon. It is a thermodynamic state where the wind formed by warm air rises (the repeller) while the swirling cold (attractor) wind descends joining together and creating a tornado, which is an equilibrium system. The mathematical modeling we present here is based on algorithms and it has been performed with Matlab code.
\end{abstract}

Keywords Dynamic System, Chaos Theory, Binary-system, Fractals, Attractor, Repeller, Matlab, Mathematical Modeling, Thermodynamic State, Tornadoes

\section{Introduction}

"Contraria non contradictoria sed complementa sunt", in the words of Niels Bohr. Current studies on tornadoes focus on a physical model. As a mathematician I seek guidelines on the nature, which can lead to physical laws. Observing natural phenomena we can deduce that the related climate behaves like 'fractal objects' in the words of Benoit Mandelbrot

Taking as reference the chaos theory, the behavior of Nature has a fractal origin; In the same way that a hurricane is an attractor and a geyseris a repeller, the only natural phenomenon we know as attractor-repeller binary system is a tornado, which is a composition of two opposing but complementary 'fractal objects'. We will try to prove this theory by presenting a mathematical model where this binary system is similar in structure and thermodynamic state to a tornado. We conclude at the end that if this is right a new line of research opens that can help us to understand

1 Opposites are not contradictory but complementary. Niels Bohr, University of Moscow . this phenomenon so devastating and which causes many human and material losses each year in most of the geography of our planet.

\section{Materials and Methods}

\subsection{Binary Systems Attractors-repellers. A Particular Case in Nature}

The Conley index theory presents a Decompositions of Invariant Sets. We begin with a definition.

Definition. Let $\mathrm{S}$ be a compact invariant set. A $\subset \mathrm{S}$ is an attractor in $\mathrm{S}$ if there exists a neighborhood $\mathrm{U}$ of $\mathrm{A}$ such that

$$
\omega(\mathrm{U} \cap \mathrm{S})=\mathrm{A}
$$

The dual repeller of $\mathrm{A}$ in $\mathrm{S}$ is

$$
\mathrm{R}:=\{\mathrm{x} \in \mathrm{S} \mid \omega(\mathrm{x}) \cap \mathrm{A}=\varnothing\}
$$

The pair $(\mathrm{A}, \mathrm{R})$ is called an attractor repeller pair descomposition of S. [1] .

This is a mathematical definition that can lead the experimental field. As mentioned in the introduction, the single repulsor attractor we know as a binary system has a natural relation which is in the origin of a tornado, where we first have a hot air rising up and at the same time there is a storm with a very low temperature making lower the air a funnel: in minutes the hot air spiral (upward spiral) meets the cold air coming down, and change the way they bind to a fractal geometry: they create a singularity, where for special conditions not yet know the internal temperature of a tornado is unknown. Therefore we cannot conclude today if it exists an adiabatic barrier or diaterma between the two 'fractal objects', but we can deduce the fractal structure that we want to analyze.

\subsection{First Repulsor. A Simple Pattern in Polar Coordinates (2D)}

Determined as a repulsive type and being the inverse of the Lorenz attractor, and from this study a model for strange 
attractors. Our model is based on plant growth, in particular is the function that describes how to grow a leaf of a deciduous tree: function 1. [2]

$$
f_{1=} \theta \cdot \sin (\theta)
$$

To see the behaviour of this function an algorithm in Matlab program is created:
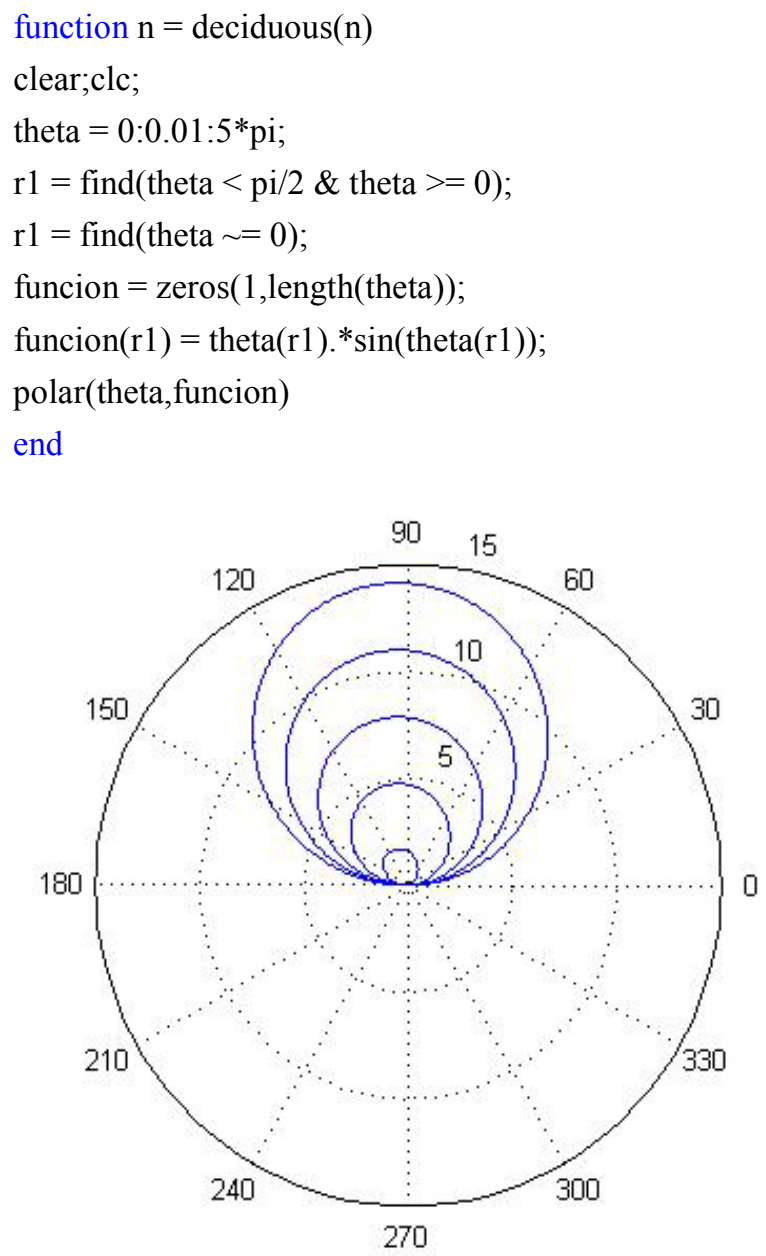

Note: with the constant theta Variable.

Figure 1. Function of the growth of a leaf of a deciduous tree

They are homothetic curves, a curve transformed by dilation that is recurrent, and resizing is repeated as it is strictly increasing. Iterations are grouped at one end.

Deriving in Matlab program we obtain function 2.

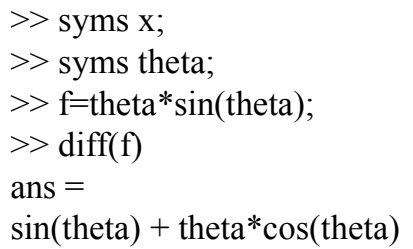

With the origin and its derivative function we will build the two fractals, the attractor and repeller.

Our parameters are the variables: From these equations and these variables an algorithm is written that passes the Matlab code [3]:
\%Authors: Gustau Camps i Valls \& Araceli Giménez Lorente

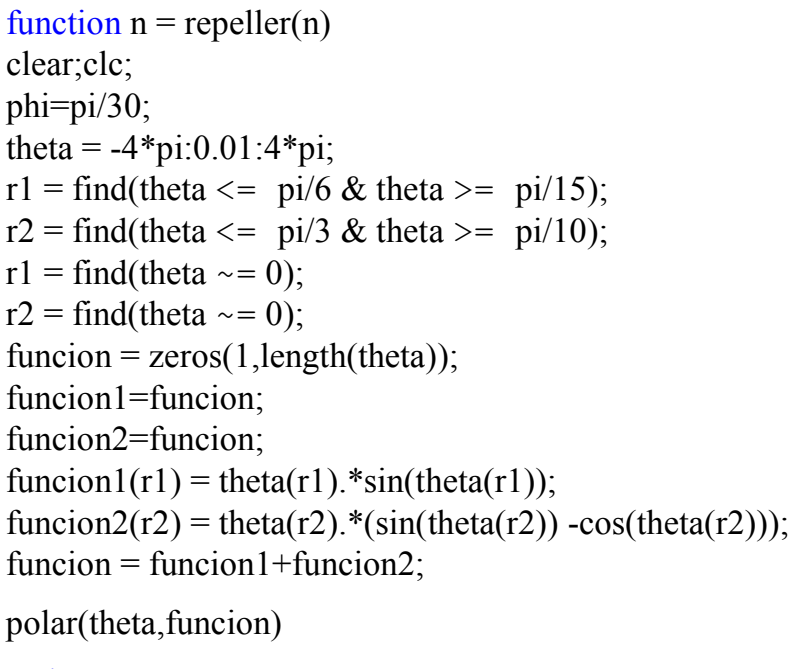

end

The inverse of the Lorenz attractor origin corresponds to the growth of a leaf of a deciduous tree. It is a finite 'fractal object', because it has been associated with a natural phenomenon which is born and dies like all living beings.

To see its growth we will study its iterations, first in polar coordinates because it is easier, and then go on to three-dimensional environment. We think the time is linked to matter-energy and it is intrinsic to its size, so each has its temporal dimension but this does not occur free; for example, the time in the first dimension would move like a pendulum but without leaving the ground, in a straight line, while in the second dimension in order to have a degree of freedom it may turn, and the third dimension of the pendulum can be lifted plane.

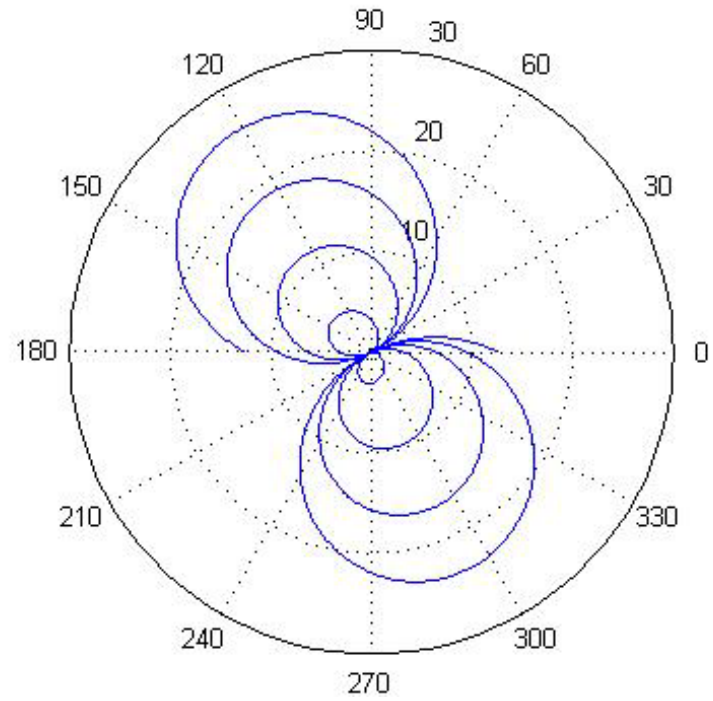

Note. Repeller in polar coordinates in Matlab

Figure 2. Reverse the Lorenz attractor, $\mathrm{L}^{-1}$

The iterations that we study here are of two types: we will visually see the growth of our repulsor for a more intuitive understanding, since we have all the time 
regarding the natural growth of a leaf from a tree, and in the end we will see if the fractals are converging or diverging.

\subsection{Iterations Repeller}

To appreciate the orbit see the repeller as increasing the iteracions of $\mathrm{n}=2$ to $\mathrm{n}=10$, where $\mathrm{n}$ corresponds to the interval at which we operate in polar coordinates:

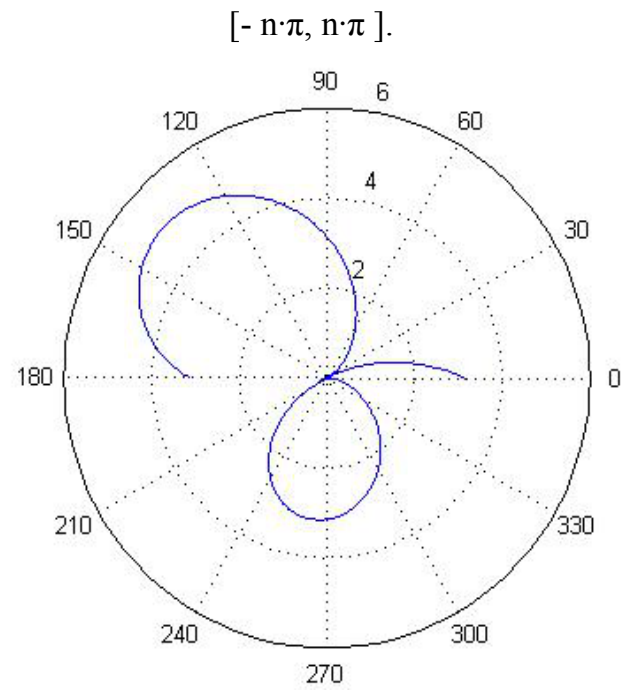

Figure 3. $\mathrm{n}=1,[-1 \cdot \pi, 1 \cdot \pi]$

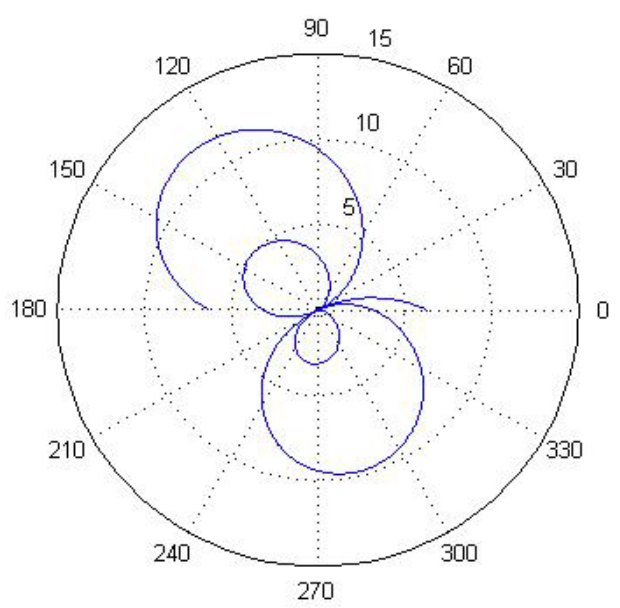

Figure 4. $\mathrm{n}=2,[-2 \cdot \pi, 2 \cdot \pi]$

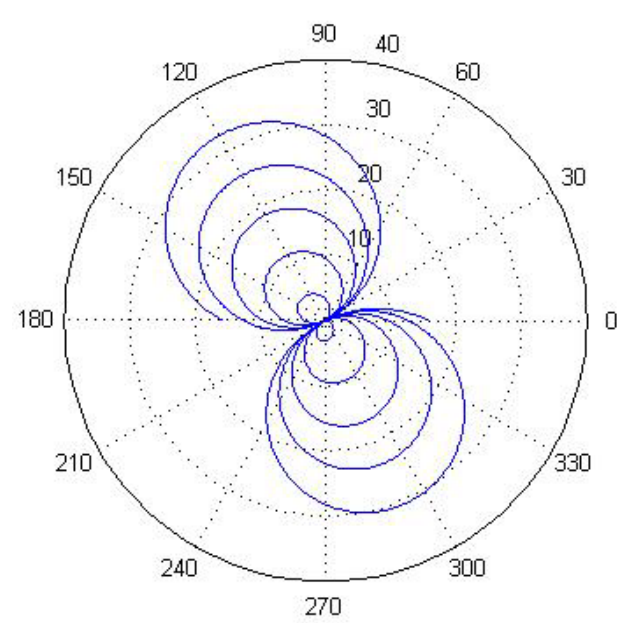

Figure 5. $\mathrm{n}=5,[-5 \cdot \pi, 5 \cdot \pi]$

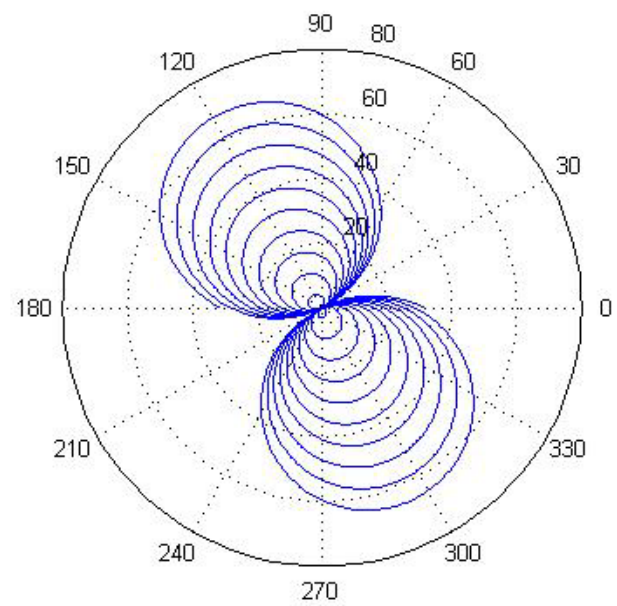

Figure 6. $\mathrm{n}=10,[-10 \cdot \pi, 10 \cdot \pi]$

We can say by looking at the images in polar coordinates that repulsor besides being a 'fractal object' has a finite characteristic that we know is similar to fractals, that is they occupy the same space. The fractal is not larger but homothetic curves are spaced less as iterations proceed.

With this pattern in polar coordinates we have a partial view of this repeller, we need to pass it to Cartesian coordinates and three-dimensional space and the natural phenomenon we find in our third dimension.

Strange attractor starting from the initial model of repeller kind. In the same equations we simulate our repulsor in $3 \mathrm{~d}$, with our algorithm in Matlab an image that will help us understand some of this generated complexity. This mathematical model corresponds to the warm wind up before a tornado funnel cloud touching the storm, and it is our repeller.

\%Author: Araceli Giménez Lorente, implementing convergence by Javier Olaya Sáez.

function $\mathrm{n}=$ repeller( $\mathrm{n}$ )

clear;

clc;

theta $=0: 0.01: 20 * \mathrm{pi}$;

$\mathrm{r} 1=$ find $($ theta $<=\mathrm{pi} / 30 \&$ theta $>=\mathrm{pi} / 3)$;

$\mathrm{r} 2=$ find $($ theta $<=\mathrm{pi} / 15 \&$ theta $>=\mathrm{pi} / 6)$;

$\mathrm{r} 3=$ find $($ theta $<=\mathrm{pi} / 2)$;

$\mathrm{r} 1=$ find $($ theta $\sim=0)$;

$\mathrm{r} 2=$ find $($ theta $\sim=0)$;

r3 = find(theta $=0)$;

$\%$ Repeller

funcion $1(\mathrm{r} 1)=$ theta $(\mathrm{r} 1) \cdot{ }^{*} \sin (\operatorname{theta}(\mathrm{r} 1))$;

funcion $2(\mathrm{r} 2)=-\cos ($ theta $(\mathrm{r} 2))$;

funcion $3(\mathrm{r} 3)=\operatorname{theta}(\mathrm{r} 1)+\operatorname{theta}(\mathrm{r} 2)+\operatorname{theta}(\mathrm{r} 3)$;

funcionbis $=\operatorname{zeros}(1$, length $($ theta $))$;

funcionbis $=$ funcion $1+$ funcion $2+$ funcion 3 ;

$\mathrm{x}=$ funcion 1 ;

$\mathrm{y}=$ funcion 2 ;

$\mathrm{z}=$ funcion 3 ; 

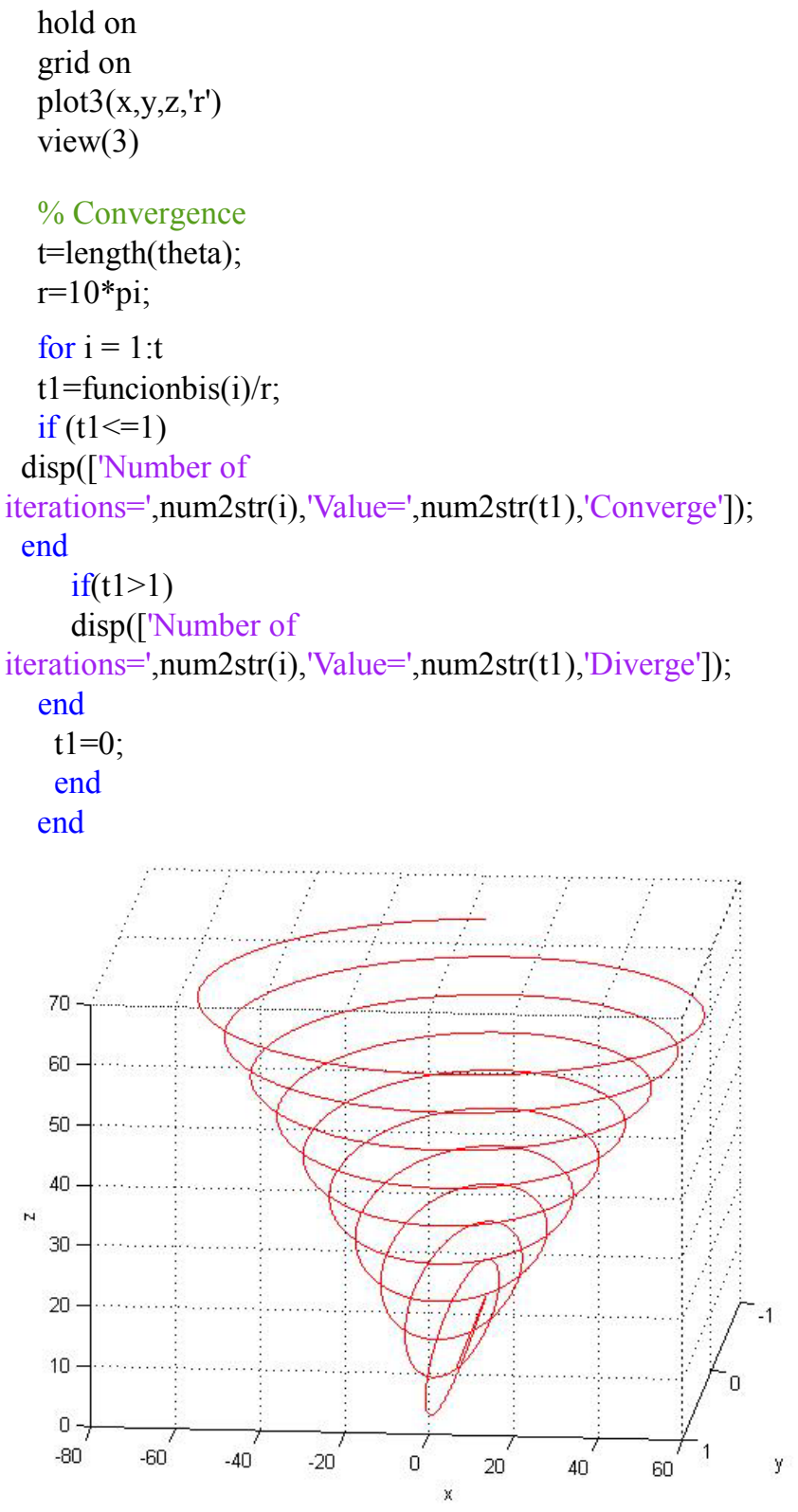

Figure 7. Repeller

Here is our strange attractor corresponding to funnel cold air coming down from the cloud before touching land before finding their way into the repeller.

\%Author: Araceli Giménez Lorente, implementing convergence by Javier Olaya Sáez.

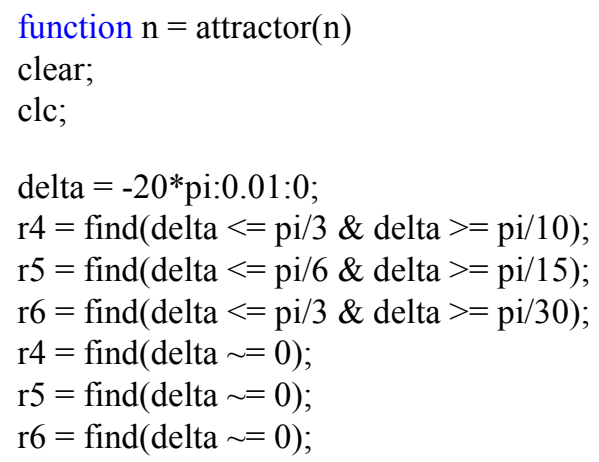

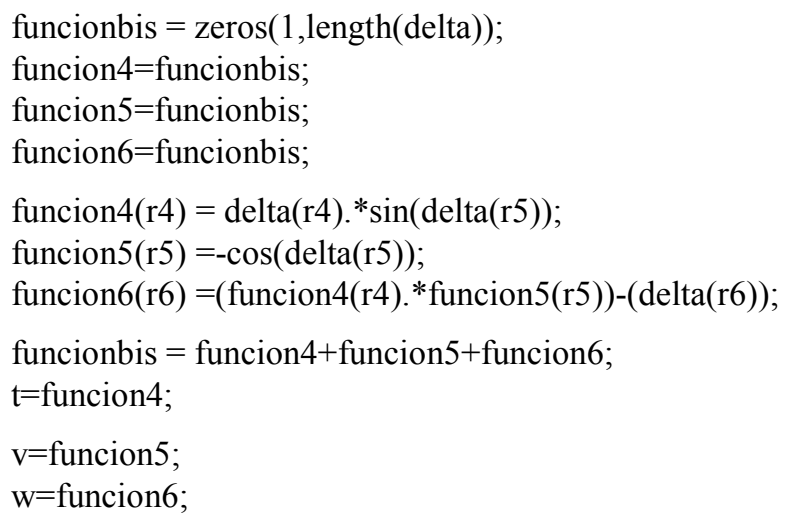

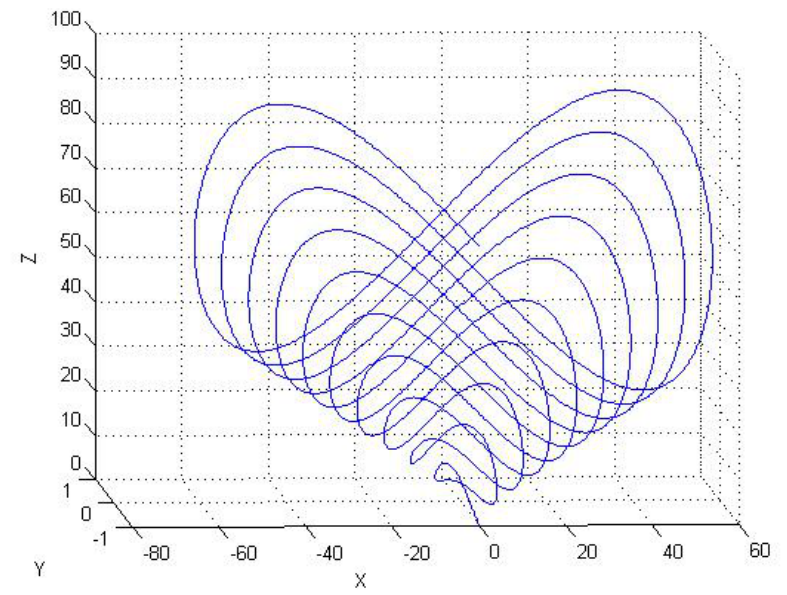

Figure 8. Attractor.

\subsection{Iterations Attractor}

The iterations that allow us to see the strange attractor orbit are as follows, for every interval $[-n \cdot \pi, n \cdot \pi]$. 


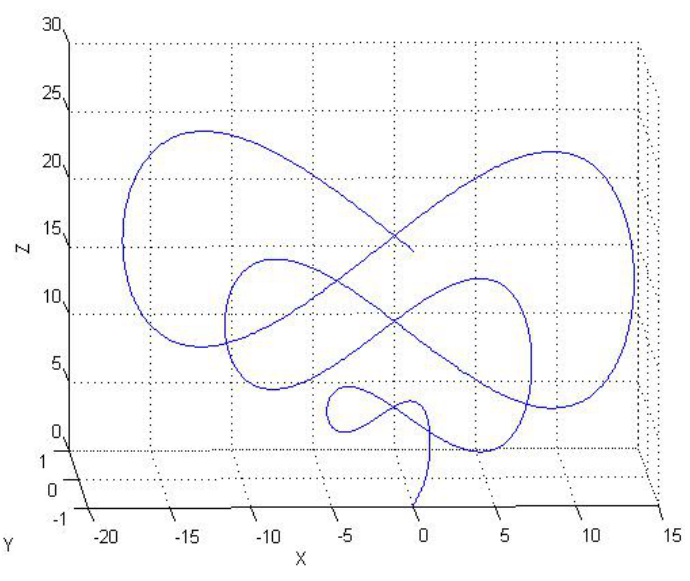

Figure 9. $\mathrm{n}=6,[-6 \cdot \pi, 0]$

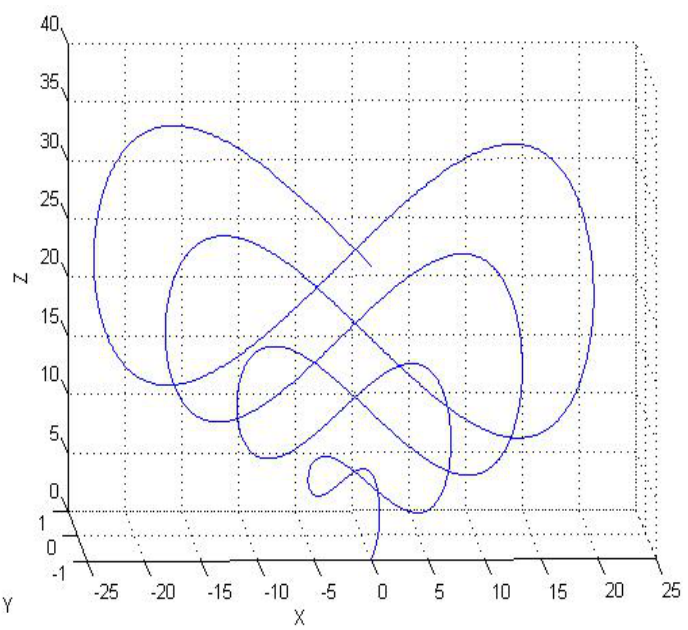

Figure 10. $\mathrm{n}=8,[-8 \cdot \pi, 0]$

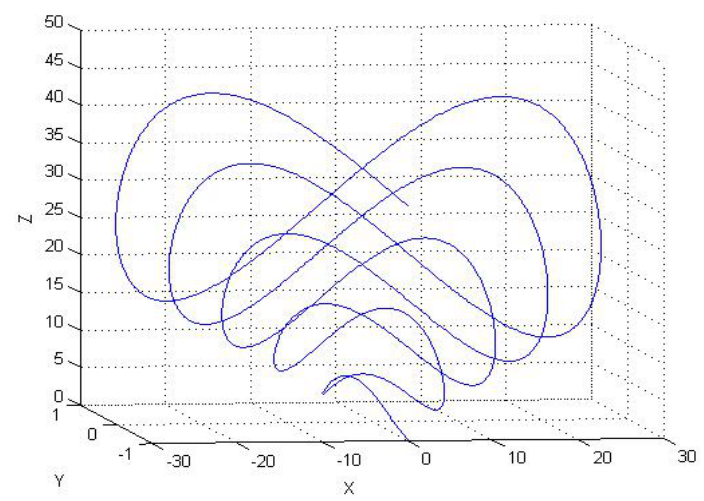

Figure 11. $\mathrm{n}=10,[-10 \cdot \pi, 0]$

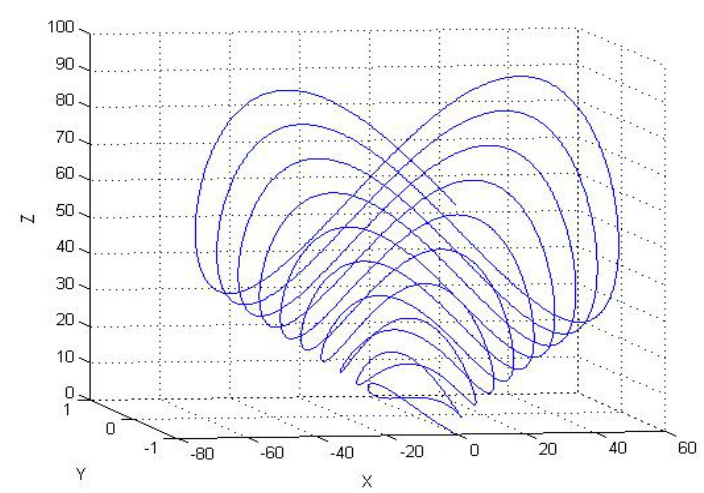

Figure 12. $\mathrm{n}=20,[-20 \cdot \pi, 0]$
As we see this strange attractor becomes more complex as they increase their iterations, defining more its structural way. The study of convergence-divergence, implemented in the algorithm written in Matlab language, comes from the following theorem. Then we will make a table of numerical values generated by the preceding programs in Matlab to study the convergence of both fractals.

Theorem 1. Consider the dynamic system $\mathbf{f}: \mathbb{R} \rightarrow \mathbb{R}$ a linear transformation defined by $\mathrm{f}(\mathrm{x})=\mathrm{rx}$, with $\mathrm{r} \neq 0$.

1. If $|r| \neq 1$ then 0 is the only fixed point of $f$, we also have:

i) if $|r|<1$, then 0 is an attractor, and the orbit of any point of $\mathrm{R}$ converges to the fixed point.

ii) if $|r|>1$, then 0 is a repeller, and the orbit of any point $\mathrm{x} \in \mathbb{R}$, with $\mathrm{x} \neq 0$., diverges to $\pm \infty$.

2. In the event that $|r|=1$, we also have:

i) if $r=1$, all points $x \in \mathbb{R}$ are fixed points and none is attractor or repeller.

ii) if $\mathrm{r}=-1,0$ is a fixed point attractor or repulsor not, and any point $x \neq 0$ is periodic of period two. [4]

\begin{tabular}{|c|c|c|c|}
\hline \multicolumn{4}{|c|}{ Binary-system } \\
\hline \multicolumn{2}{|c|}{ Attractor } & \multicolumn{2}{c|}{ Repeller } \\
\hline $\begin{array}{c}\text { Number of } \\
\text { Iterations }\end{array}$ & $\begin{array}{c}\text { Convergence if } \\
\mathrm{x}<1\end{array}$ & $\begin{array}{c}\text { Number of } \\
\text { Iterations }\end{array}$ & $\begin{array}{c}\text { Divergence if } \\
\mathrm{x}>1\end{array}$ \\
\hline $\mathrm{n}=1$ & $-0.98408<1$ & $\mathrm{n}=1$ & $2.9682>1$ \\
\hline $\mathrm{n}=2$ & $-0.98393<1$ & $\mathrm{n}=2$ & $2.9791>1$ \\
\hline $\mathrm{n}=3$ & $-0.98377<1$ & $\mathrm{n}=3$ & $2.9901>1$ \\
\hline $\mathrm{n}=4$ & $-0.9836<1$ & $\mathrm{n}=4$ & $3.0011>1$ \\
\hline $\mathrm{n}=5$ & $-0.98343<1$ & $\mathrm{n}=5$ & $3.0121>1$ \\
\hline $\mathrm{n}=6$ & $-0.98325<1$ & $\mathrm{n}=6$ & $3.023>1$ \\
\hline $\mathrm{n}=7$ & $-0.98305<1$ & $\mathrm{n}=7$ & $3.034>1$ \\
\hline $\mathrm{n}=8$ & $-0.98284<1$ & $\mathrm{n}=8$ & $3.045>1$ \\
\hline $\mathrm{n}=9$ & $-0.98261<1$ & $\mathrm{n}=9$ & $3.056>1$ \\
\hline $\mathrm{n}=10$ & $-0.98235<1$ & $\mathrm{n}=10$ & $3.067>1$ \\
\hline $\mathrm{n}=20$ & $-0.97796<1$ & $\mathrm{n}=20$ & $3.1769>1$ \\
\hline $\mathrm{n}=30$ & $-0.96825<1$ & $\mathrm{n}=30$ & $3.2858>1$ \\
\hline $\mathrm{n}=40$ & $-0.9507<1$ & $\mathrm{n}=40$ & $3.3927>1$ \\
\hline $\mathrm{n}=50$ & $-0.92321<1$ & $\mathrm{n}=50$ & $3.4967>1$ \\
\hline $\mathrm{n}=60$ & $-0.88421<1$ & $\mathrm{n}=60$ & $3.5967>1$ \\
\hline $\mathrm{n}=70$ & $-0.83273<1$ & $\mathrm{n}=70$ & $3.6919>1$ \\
\hline $\mathrm{n}=80$ & $-0.7685<1$ & $\mathrm{n}=80$ & $3.7813>1$ \\
\hline $\mathrm{n}=90$ & $-0.69192<1$ & $\mathrm{n}=90$ & $3.864>1$ \\
\hline $\mathrm{n}=100$ & $-0.60415<1$ & $\mathrm{n}=100$ & $3.9394>1$ \\
\hline $\mathrm{n}=200$ & $0.26969<1$ & $\mathrm{n}=200$ & $4.1743>1$ \\
\hline $\mathrm{n}=300$ & $-1.0569<1$ & $\mathrm{n}=300$ & $3.4824>1$ \\
\hline $\mathrm{n}=400$ & $-2.1141<1$ & $\mathrm{n}=400$ & $2.5566>1$ \\
\hline $\mathrm{n}=500$ & $-1.5589<1$ & $\mathrm{n}=500$ & $2.3533>1$ \\
\hline $\mathrm{n}=600$ & $-0.90059<1$ & $\mathrm{n}=600$ & $3.1974>1$ \\
\hline $\mathrm{n}=700$ & $-0.73838<1$ & $\mathrm{n}=700$ & $4.4372>1$ \\
\hline $\mathrm{n}=800$ & $0.10704<1$ & $\mathrm{n}=800$ & $5.01>1$ \\
\hline $\mathrm{n}=900$ & $-0.18305<1$ & $\mathrm{n}=900$ & $4.4291>1$ \\
\hline $\mathrm{n}=1000$ & $-1.6853<1$ & $\mathrm{n}=1000$ & $3.2749>1$ \\
\hline & $-0.31406<1$ & $\mathrm{n}=2000$ & $6.3827>1$ \\
\hline & $-0.96295<1$ & $\mathrm{n}=3000$ & $3.9251>1$ \\
\hline & $-0.48418<1$ & $\mathrm{n}=3142$ & $5.9558>1$ \\
\hline
\end{tabular}




\subsection{Modeling of a Tornadoes}
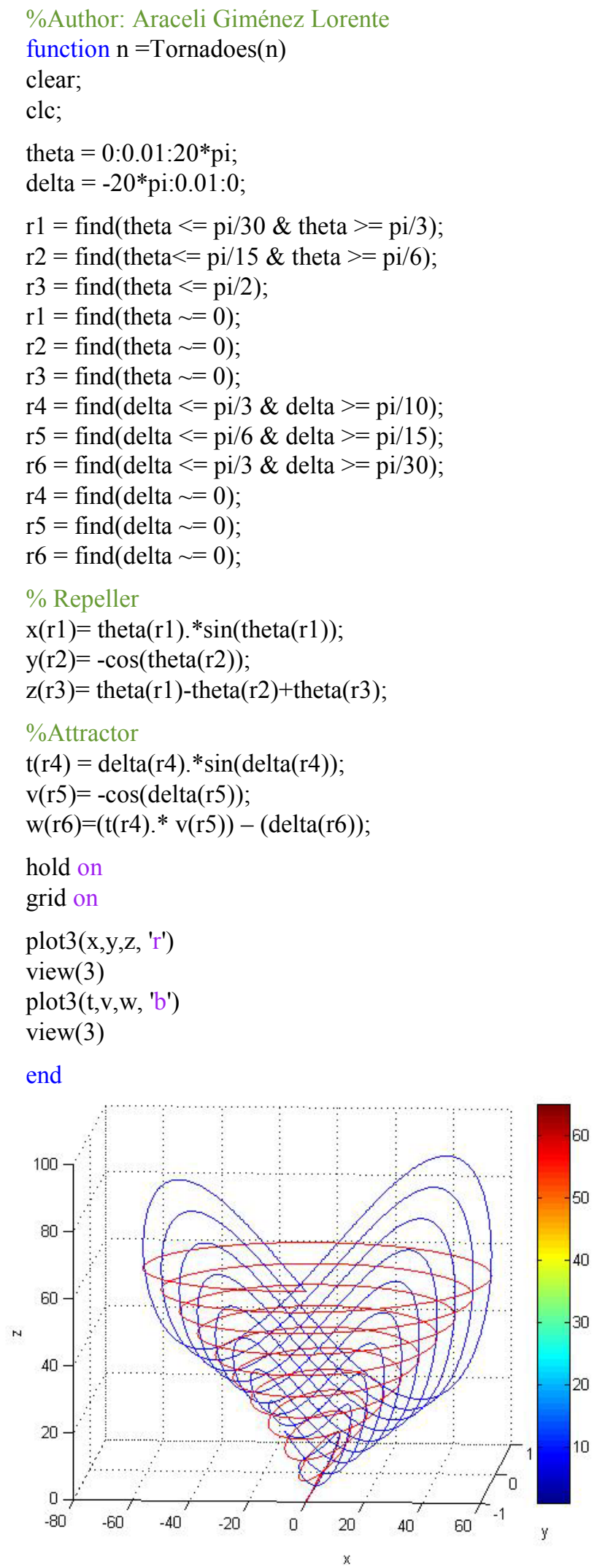

Note. Here we can see the blue attractor and repeller red color associated with a thermodynamic state.

Figure 13. Modelling of a Tornadoes
Here we have our mathematical modeling of a tornado as the composition of the previous two fractals. The attractor-repeller binary system was originally proposed.

\section{Discussion}

The vortex we have in a tornado is in cyclostrophic balance [5] and it is possible because wind is the result of balance between the baric force and centrifugal force, at this time the Coriolis component is negligible. This balance occurs when the pressure force and centrifugal force are opposite as in a storm.

In the center of the tornadoes atmospheric pressure is 100 millibars lower than that of its surroundings. With maximum winds of $650 \mathrm{~km} / \mathrm{h}$ is the most aggressive atmospheric phenomenon. There is a temperature difference between the repeller's hot and the attractor corresponding to cold air, as an initial hypothesis for future work.

The zeroth law of thermodynamics is to define the relationship systems in thermal equilibrium: if $\mathrm{A}$ and $\mathrm{B}$ are separately in thermal equilibrium with $\mathrm{C}$, also are in thermal equilibrium with each other (transitive property)" [5].

$$
\text { If } \mathrm{T}_{\mathrm{A}}=\mathrm{T}_{\mathrm{B}} \text { and } \mathrm{T}_{\mathrm{B}}=\mathrm{T}_{\mathrm{C}} \rightarrow \mathrm{T}_{\mathrm{A}}=\mathrm{T}_{\mathrm{C}}
$$

We have that as attractors-repellers binary systems are stable thermodynamically destabilizing one of the two systems. Tornadoes would be in a situation of imbalance and evaporate or collapse.

\section{Acknowledgements}

I thank Ph.Doctor Vicente Caselles Miralles for believing in my potential as a scientist and as a mentor to support me, thank Ph. Doctor Gustavo Camps i Valls because I introduced in Matlab programming that allowed me to do my math modeling, and would also like to thank my friend Javier Olaya Sáez., who is also physical, your help with programming.

And thanks to NOAA for all your information available on the website have helped me understand this atmospheric phenomenon.

\section{REFERENCES}

[1] Mischaikow,Konstantin; Mrozek,Marian. Conley Index Theory. Preprint. p. 7. 1999.

[2] Borrás Veses, Eliseo; Moreno Gómez, Pilar; Nomdedeu Moreno, Xaro. Ritmos. Matemáticas e imágenes. Nivola.Madrid, pp.70-71. 2002.

[3] Giménez Lorente, Araceli. Los Estados Cuánticos de la Materia-Energía. E.A.E, GmbH \&Co. Saarbrücken, (Deutschland), pp. 55-57.2012.

[4] Arrollo, Aubin; Seade, José. Sistemas dimámicos discretos: 
Una introducción. Unidad Cuernavaca, Instituto de Matemáticas, Universidad Nacional Autónoma de México, (México), p. 4. 2011.

[5] Wallace, John M.; Hobbs, Peter V Atmospheric Science. An
Introductory Survey. Academic Press is an imprint of Elsevier, p.374. 2006.

[6] Aguilar Peris, José. Curso de Termodinámica. Ed. Alhambra Universidad, p. 19. Madrid 1989. 